\title{
Wahrnehmung und Darstellung von Räumen der Kindheit
}

Rezension zu Jürgen Hasse / Verena Schreiber (Hg.) (2019): Räume der Kindheit. Ein Glossar. Bielefeld: transcript.

Abb. 1 Titel des Buches (Quelle: transcript-Verlag)

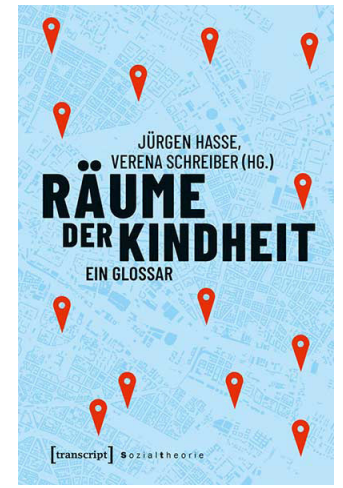

„Wie in den Märchenwald, so geht das Kind in die Welt hinein, angelockt von allen Reizen des Neuen, Unbekannten und Wohlgesinnten. Denn wohlgesinnt und unerschöpflich scheint die Welt. Nicht sinnlos, aber auch nicht ,bekannt': es ist noch alles möglich und deshalb gibt es noch keinen Un-sinn.“ (Langeveld 1968: 142)

Im kindlichen Spiel drückt sich die besondere Qualität des In-der-WeltSeins von Kindern aus. Für Erwachsene scheinbar Triviales lädt dazu ein, immer wieder neu erschlossen und mit Bedeutung versehen zu werden. Ein einfacher Tisch dient dann nicht nur dazu, gedeckt und als Essplatz genutzt zu werden, sondern fordert unter anderem dazu auf, unter ihn zu kriechen, um als Versteck, Schutzraum oder Höhle zu fungieren. Dem Erwachsenwerden halten solche Lesarten in der Regel nicht stand. Es ist - mit Martinus Langeveld gesprochen - nicht mehr alles möglich; der Märchenwald wird im Extremfall zum Forst. Aus dieser Perspektive sind die Anliegen und die Sinngebung von Kindern keine naiven Vorformen korrekter Sichtweisen von Erwachsenen. Zugleich wird die Art und Weise, wie Kind und Welt zueinander in Beziehung treten, durch Sozialisationsprozesse geprägt und immer auch auf bestimmte Weise gezähmt und geformt. Und doch: Wie sich uns im kindlichen Spiel bestimmte Orte und Räume zeigten, zu was sie uns einluden, wie wir sie uns erschlossen und aushandelten, bleibt stets Teil unseres Erfahrungshorizonts - auch wenn sich bestimmte räumliche Erfahrungsschichten zunehmend abschatten und in Vergessenheit geraten.

Das von Jürgen Hasse und Verena Schreiber herausgegebene Glossar Räume der Kindheit tritt an, sich Prozessen der Raumwahrnehmung und -aneignung von Kindern zu widmen und dabei zugleich denjenigen Orten und Räumen Aufmerksamkeit zu schenken, die in der Kindheitsforschung am Rande der üblichen Wahrnehmung liegen. So finden sich im Glossar nicht nur Einträge, die in der Diskussion eindeutig als Räume der Kindheit chiffriert sind, wie „Kindergarten“ oder „Schule“. Es werden auch Begriffe erkundet, die sich erst auf den zweiten Blick als Räume der Kindheit zu erkennen geben, wie „Karussell“, „Lunchbox“ oder „Pferderücken“. Den 
Herausgeber*innen geht es darum, liminale Räume in den Blick zu rücken, das heißt „das Übersehene und Vergessene, das scheinbar Marginale und Gewöhnliche (wieder) denkwürdig zu machen“(Hasse/Schreiber 2019b: 12).

Der große Gewinn dieses Glossars besteht darin, dass es nicht den Versuch unternimmt, Räume der Kindheit lückenlos und lexikalisch zu vermessen. Es bleibt - das ist ausdrücklich so angelegt und gewollt (vgl. Hasse/Schreiber 2019b: 13) - vieles offen und gibt sich nicht ohne Weiteres preis. So ist beim Aufschlagen des Buches konsequenterweise auch keine Systematik bei der Begriffsauswahl erkennbar und beispielsweise zwischen „Adoptiert werden“, „Kinderzimmer“ und „Utopie“wohl auch nicht sinnfällig. Insgesamt haben die Herausgeber*innen in einem Register die beachtliche Anzahl von 63 Räumen der Kindheit zusammengetragen. Auch wenn sie mit der Perspektivität und den Leerstellen des Glossars bereits zu Beginn kritisch umgehen und auch einige Einblicke in den Findungsprozess der Begriffe geben, bleiben beim Lesen doch auch einige spannende Fragen offen. Warum wurde beispielsweise manches wieder verworfen, ,was doch zunächst so vielversprechend klang“ (Hasse/Schreiber 2019b: 13)? Woran wurde also festgemacht, ob ein Begriff passend für das Glossar ist?

Den von den Herausgeber*innen festgelegten Begriffen spürten insgesamt 67 Autor*innen aus unterschiedlichen Sozial-, Kultur- und Geisteswissenschaften, wie Pädagogik, Didaktik und Hebammenwissenschaft in kompakten Texten zu je sechs bis acht Seiten nach. Sowohl theoretisch und methodisch, als auch strukturell und sprachlich liegen also sehr unterschiedliche Glossareinträge vor. Für die kritische Stadtforschung ergeben sich hieraus eine Reihe unterschiedlicher Bezüge und Anknüpfungspunkte, wobei diese in den einzelnen Beiträgen unterschiedlich konkret herausgestellt werden. Aufgrund der gebotenen Kürze können hier nur zwei Beispiele genannt werden, die einerseits die Spannbreite des Glossars verdeutlichen und andererseits dessen Potenzial für die Stadtforschung skizzieren:

Im Eintrag „Kindergarten“ verdeutlicht die Erziehungs- und Bildungswissenschaftlerin Sabine Bollig ausgehend von der aktuellen Namensgebung Berliner Kindertagesstätten sowie den reformpädagogischen Vorstellungen Friedrich Fröbels vom Kindergarten als „Garten-Paradies“ den neoliberalen Gehalt aktueller, politisch geforderter frühkindlicher Bildung in Kindertagesstätten. Diese solle zunehmend die „employability und Demokratiefähigkeit [...] des nachwachsenden Humankapitals im Horizont sich zunehmend globalisierender und digitalisierender Wirtschafts- und Arbeitsmärkte und sich pluralisierender Gesellschaften“ (Bollig 2019: 161) sicherstellen. Für die Stadtforschung ist in diesem Zusammenhang unter anderem die Spur im Beitrag interessant, wie Kindertagesstätten in der neoliberalen Stadt konkret „als Orte der Reproduktion (und nicht der Kompensation) sozialer Ungleichheiten“ (ebd.: 164) fungieren.

Der Kulturanthropologe Oliver Müller widmet sich in seinem Eintrag dem „Kiosk“ in seiner lebensweltlichen Bedeutung für Kinder und Jugendliche, aber auch seinen Merkmalen als „Raumtyp Kiosk“ (Müller 2019: 187, Hervorhebung im Original). Müller versteht Kioske als Gegen-Orte und, aus Sicht von Kindern, als „explorative Fenster zur Welt“ (ebd.), in denen erste Erfahrungen des sozialräumlichen Miteinanders gemacht sowie sozioökonomische Praktiken eingeübt und verinnerlicht werden. Insofern ist der 
Kiosk als „halböffentlicher Schwellenraum der Stadt“ (Müller 2019: 188), der als einer der ersten Stadträume überhaupt von Kindern „auf eigene Faust erkundet“ (ebd.) wird, eben auch eine lohnenswerte Spur für die kritische Stadtforschung: Wie werden hier welche urbanen Praktiken und (Ungleichheits-)Strukturen fortlaufend (re-)produziert?

Das essayistische Vorgehen des Glossars ist begrüßenswert und überaus ertragreich, da es dem Umstand Rechnung trägt, dass die Einträge einen bestimmten Raum der Kindheit niemals erschöpfend einfangen können, sondern stets nur bestimmte Perspektiven zur Darstellung bringen. Der Versuch, bruchlose Lexikoneinträge zu Räumen der Kindheit zu verfassen, müsste angesichts des Gegenstands auch unweigerlich scheitern. Die Glossareinträge zeugen also ebenso von begrifflich-analytischer Betrachtung (beispielsweise im Eintrag „Brennpunkt“) wie von Beobachtungen des kindlichen Spiels in einer Kita (etwa im Eintrag „Zaun“), von Gesprächen mit Nachbarskindern (beispielsweise im Eintrag „Fußboden“), von autobiografischen Erzählungen (etwa im Eintrag „Höhle“) oder von Auseinandersetzungen mit literarischen Darstellungen (beispielsweise im Eintrag „Buch“). Die Herausgeber*innen beschreiben diese Vielfalt des Glossars einleitend treffend als ein buntes Bücherregal, das zum Stöbern, Weiterlesen und Herstellen von Verbindungen einlädt (Hasse/Schreiber 2019b: 9 f.). Dennoch wünscht man sich als Leser*in an manchen Stellen eine deutlichere Sortierung dieses Bücherregals, beispielsweise durch eine konkretere Orientierung an unterschiedlichen Ansätzen der Kindheitsforschung (vgl. Alanen 2014) oder einer konsequenten Abgrenzung von dieser. Als Fixpunkt des Glossars ist (neben dem Gegenstand „Räume der Kindheit“) in erster Linie eine „hermeneutische Wachsamkeit“ (van Manen 1990: 96, Übers. d. A.) gegenüber den jeweils erkundeten Situationen und Räumen festzuhalten. Die einzelnen Beiträge oszillieren stets gekonnt zwischen lebensweltlicher Beschreibung und wissenschaftlicher Reflexion und überzeugen aufgrund ihrer Prägnanz. Das Verhältnis zwischen Beschreibung und Interpretation verläuft dabei fließend und auch in Abhängigkeit von der jeweiligen Forscher*innenbiografie mit unterschiedlicher Schwerpunktsetzung.

Das Glossar richtet sich gleichermaßen an Fachwissenschaftler*innen und Fachdidaktiker*innen aus den Sozial-, Kultur- und Geisteswissenschaften, ist aber durchaus auch für Nicht-Fachleute von Interesse, da der Gegenstand - Räume der Kindheit - auf unser aller Biografien verweist. Zur Lektüre bringt jede* $\mathrm{r}$ eigene Erfahrungen mit dem Gegenstand des Buches mit - was überaus gewinnbringend ist. Denn sich als Leser*in auf Betrachtungen dieser vermeintlich gut bekannten alltäglichen Orte und Räume einzulassen, die allzu oft am Rande der üblichen Wahrnehmung liegen, birgt das Potenzial, sich an die Räume der eigenen Kindheit zu erinnern, sich also eigener Erfahrungen bewusst zu werden und andere Erfahrungen nachzuvollziehen, der eigenen Eingebundenheit in Blickregime nachzuspüren und im günstigsten Fall eingeschliffene Sichtweisen irritieren zu lassen. Damit hält die Lektüre des Glossars auch wertvolle Impulse für die eigene Forschungspraxis bereit. Die Glossareinträge zeigen eindrücklich auf, wie das Sich-Einlassen auf Lebenswelten und -wirklichkeiten von Kindern aus einer Erwachsenenperspektive gelingen kann - mitsamt all der Implikationen, die dieses Vorgehen mit sich bringt. Denn es fällt auf, dass die Stimmen von 
Kindern im Glossar nur selten unmittelbar zur Sprache kommen (beispielsweise im Eintrag „Unterwegssein“, in dem die Geographin Michaela Schier theoretische Reflexion und Interviewausschnitte zur Alltagsmobilität von Kindern auf überzeugende Weise miteinander verzahnt). Diese Leerstelle des Glossars verwundert etwas, da es durchaus wünschenswert und auch im Sinne einer partizipativen Kindheitsforschung wäre, sich dem kindlichen Erleben von Stadt nicht ausschließlich retrospektiv, aus der eigenen Erfahrung und als Forscher*in zu widmen, sondern dem Erleben von Kindern als Expert*innen mehr Raum zu geben.

Zusammengefasst legen die Herausgeber*innen mit dem Glossar Räume der Kindheit kein einfaches Nachschlagewerk vor, sondern präsentieren Ergebnisse einer Suche nach Möglichkeiten der Beschreibung und kritischen Reflexion von Räumen der Kindheit. Der transdisziplinäre Zugang weitet den Blick auch über etablierte kindheitssoziologische Perspektiven auf Räume der Kindheithinaus. Erstellt diesen unter anderem philosophische, geographische und pädagogische Sichtweisen zur Seite. Diese kaleidoskopartige Struktur ist - ebenso wie die essayistische Form des Buches - überaus bereichernd und zugleich im Kontext der Kindheitsforschung ein Alleinstellungsmerkmal.

Gegen Ende sei der erste Satz des Glossars zitiert: „Räume der Kindheit können überall sein.“(Hasse/Schreiber 2019b: 9) Dem sei hinzugefügt: Man muss nur lernen, (erneut) auf sie aufmerksam zu werden. Für die kritische, stadtbezogene Kindheitsforschung bietet der Band das Potenzial, den Blick für solche theoretischen Perspektiven sowie für solche Räume der Kindheit zu öffnen, denen in der Kindheitsforschung allzu oft keine Beachtung geschenkt wird. Aber auch zur Reflexion derselben in ihrer Bedeutung für das räumliche Erleben und räumliche Aneignungspraktiken von Kindern leistet das Buch einen wichtigen Beitrag und lädt zugleich ein, die Glossareinträge als Spuren zu verstehen, die es aufzugreifen, weiterzudenken und zum Gegenstand kritischer Reflexion zu machen gilt.

Dieser Artikel wurde durch den Open-Access-Publikationsfonds der Universität Graz gefördert.

\section{Autor_innen}

Fabian Pettig ist Fachdidaktiker. Er forscht zu Gelingensbedingungen kritischer geographischer Bildung im Anthropozän sowie einer Kultur der Digitalität.

fabian.pettig@uni-graz.at

\section{Literatur}

Alanen, Leena (2014): Childhood and intergenerationality. Toward an intergenerational perspective on child well-being. In: Asher Ben-Arieh / Ferran Casas / Ivar Frønes / Jill E. Korbin (Hg.), Handbook of child well-being. Theories, methods and policies in global perspective. Dordrecht u. a.: Springer, 131-160.

Bollig, Sabine (2019): Kindergarten. In: Jürgen Hasse / Verena Schreiber (Hg.), Räume der Kindheit. Ein Glossar. Bielefeld: transcript, 159-165.

Hasse, Jürgen / Schreiber, Verena (Hg.) (2019a): Räume der Kindheit. Ein Glossar. Bielefeld: transcript. 
Hasse, Jürgen / Schreiber, Verena (2019b): Einleitung. In: Jürgen Hasse / Verena Schreiber (Hg.), Räume der Kindheit. Ein Glossar. Bielefeld: transcript, 9-14.

Langeveld, Martinus Jan (1968): Studien zur Anthropologie des Kindes. Tübingen: Max Niemeyer.

van Manen, Max (1990): Researching lived experience. Human science for an action sensitive pedagogy. New York: State University of New York Press.

Müller, Oliver (2019): Kiosk. In: Jürgen Hasse / Verena Schreiber (Hg.), Räume der Kindheit. Ein Glossar. Bielefeld: transcript, 185-190.

Schier, Michaela (2019): Unterwegssein. In: Jürgen Hasse / Verena Schreiber (Hg.), Räume der Kindheit. Ein Glossar. Bielefeld: transcript, 342-347. 